\title{
A Simple Way for Implementing Extraction Columns of Infinite Height in Flowsheet Simulators
}

\author{
M. Kaul ${ }^{\text {a, b }}$, H. Hasse ${ }^{\text {a }}$, J. Burger ${ }^{\text {c, * }}$ \\ a University of Kaiserslautern, Laboratory of Engineering Thermodynamics, \\ Erwin-Schrödingerstraße 44, 67663 Kaiserslautern, Germany \\ ${ }^{b}$ Current affiliation: BASF SE, 67056 Ludwigshafen, Germany \\ c Technical University of Munich, Campus Straubing, Chair for Chemical Process \\ Engineering, Schulgasse 16, 94315 Straubing, Germany
}

\begin{abstract}
A surrogate model to implement an extraction column of infinite height in process simulation software is presented. The model consists of three decanters and a few specifications which are easy to implement in commercial process simulators. Using the model, the minimum solvent flow rate and the limiting product compositions are determined. In an example, the surrogate model for the extraction column is combined with standard unit operation models for describing an extraction process with a distillative solvent recycle.
\end{abstract}

Keywords: Surrogate model, slope curve method, pinch behavior, extraction column

\section{Introduction}

Pinch-based apparatus models play an important role in conceptual process design. The core assumption is that the apparatus is of infinite size, which leads to the assumption of an infinite number of theoretical stages in distillation, absorption, or extraction models. In the limiting case of infinite height, the apparatuses optimal separation performance $[1,2,3]$. The assumption of an infinite number of stages leads to a small set of mathematical equations, usually

*Corresponding author. Tel: +49 9421187275

Email adress: burger@tum.de (J. Burger) 
called the pinch equations $[4,5]$, that describe the steady-state behavior of the apparatus. In the area of distillation, analysis of the pinch equations has lead to a vast number of analysis methods and short-cut models that have been implemented in software, examples are the boundary value method [6] which is implemented in the simulator Aspen Plus [7], the rectification body method $[8,9]$, or the $\infty / \infty$-analysis tools by Ryll et al. $[3,10,11]$.

The pinch behavior of counter-current extraction columns is by far less studied than the one for distillation columns. Minotti et al. [4] derived the pinch equations for extraction columns and showed how to use them in design tasks [12]. The pinch equations are a set of nonlinear equations and their solutions yields the products of an extraction column of infinite height for given input streams of the column. Since the pinch equations have several, partly nonphysical solutions, Minotti et al. [4] used a geometrical solution method which is easy to initialize but limited to systems with up to four components [13]. Another geometrical solution method was presented by Burger et al. [14] who proposed to transform the concentration space into a special diagram, the slope diagram, to determine the location of the pinch and the column's product compositions. A short-cut model based on pinch equations for extraction columns for multi component mixtures was presented by Redepenning et al. [13].

The aforementioned methods require specialized software or a custom-made code implementation which hampers their applicability in industrial practice. The presents work demonstrates how to implement the pinch equations of extraction columns into standard process simulators. The goal is to have an implementation of an infinitely high column using standard unit operation models. The resulting model is called surrogate model in the following as it replaces the infinite height extraction column in the flowsheet simulation. Using that surrogate model in the environment of the process simulator enables the process engineer to integrate and solve the pinch equations in a common workflow without further ado: i.e. the built-in thermodynamic models of the simulator are used, and the surrogate model of the infinite height extraction column can be combined with other arbitrary unit models in a flowsheet. 
Bonet et al. [15] pursued a similar goal and proposed to use a single decanter as a surrogate model of the column. Special schemes were used for the implementation and evaluation in a standard process simulator. However, their approach yields only an approximation of the solution for the model of the column of infinite height and the combination of the decanter with other unit operation models is difficult since the product streams of the emulated column are not directly available for connection with other units. These limitations are overcome in the present work.

After briefly reviewing the fundamentals of the pinch equations in extraction columns in the next section, the surrogate model and its implementation are presented. Finally, an example is discussed in which the surrogate model was implemented in the commercial process simulator Aspen Plus [7] and combined with other unit operation models to a full flowsheet.

\section{Fundamentals}

\subsection{Pinch equations for extraction columns}

The nomenclature that is used here for describing an extraction column with $N$ equilibrium stages is depicted in Figure 1. The streams entering the column are the feed $\mathrm{F}$ at stage $N$ and the solvent $\mathrm{S}$ at stage 1. The streams leaving the column are the extract $\mathrm{E}^{N}$ at stage $N$ and the raffinate stream $\mathrm{R}^{1}$ at stage 1. At every stage $i=1 \ldots N$, the streams $\mathrm{E}^{i}$ and $\mathrm{R}^{i}$ that leave the stage are in liquid-liquid equilibrium (LLE). 


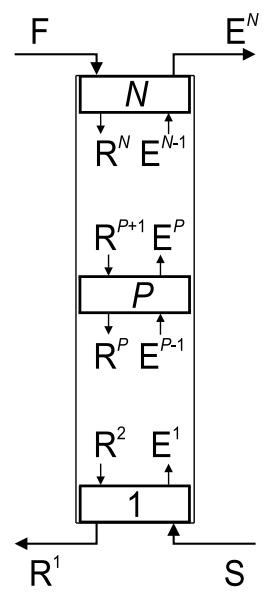

Figure 1: Extraction column of infinite height with the feed $\mathrm{F}$, the solvent $\mathrm{S}$, the extract $\mathrm{E}^{N}$, and the raffinate $\mathrm{R}^{1}$. Stage $P$ marks the pinch.

For infinitely high columns, there must be at least one pinch, where the driving force for separation become infinitesimally small and the compositions of the counter-current phases do not change anymore between adjacent stages. This is the case for stage $P$ in Figure 1. In the spirit of Minotti et al. [4] and Redepenning et al. [13], the so-called pinch equations relating the streams from Figure 1 are giving in the following.

On the stages $1, P, N$ the material balances hold:

$$
\begin{gathered}
\mathbf{x}^{\mathrm{R}, 1} \cdot \dot{n}^{\mathrm{R}, 1}-\mathbf{x}^{\mathrm{S}} \cdot \dot{n}^{\mathrm{S}}=\mathbf{x}^{\mathrm{R}, 2} \cdot \dot{n}^{\mathrm{R}, 2}-\mathbf{x}^{\mathrm{E}, 1} \cdot \dot{n}^{\mathrm{E}, 1} \\
\mathbf{x}^{\mathrm{R}, P} \cdot \dot{n}^{\mathrm{R}, P}-\mathbf{x}^{\mathrm{E},(P-1)} \cdot \dot{n}^{\mathrm{E},(P-1)}=\mathbf{x}^{\mathrm{R},(P+1)} \cdot \dot{n}^{\mathrm{R},(P+1)}-\mathbf{x}^{\mathrm{E}, P} \cdot \dot{n}^{\mathrm{E}, P} \\
\mathbf{x}^{\mathrm{R}, N} \cdot \dot{n}^{\mathrm{R}, N}-\mathbf{x}^{\mathrm{E},(N-1)} \cdot \dot{n}^{\mathrm{E},(N-1)}=\mathbf{x}^{\mathrm{F}} \cdot \dot{n}^{\mathrm{F}}-\mathbf{x}^{\mathrm{E}, N} \cdot \dot{n}^{\mathrm{E}, N}
\end{gathered}
$$

Therein, $\mathbf{x}^{j}$ denotes the mole fraction vector of stream $j$ and $\dot{n}^{j}$ its total molar flow rate. From the LLE of streams leaving the stages, it follows:

$$
0=f_{\mathrm{LLE}}\left(\mathrm{x}^{\mathrm{E}, 1}, \mathrm{x}^{\mathrm{R}, 1}\right)
$$




$$
\begin{aligned}
& 0=f_{\mathrm{LLE}}\left(\mathbf{x}^{\mathrm{E}, P}, \mathbf{x}^{\mathrm{R}, P}\right) \\
& 0=f_{\mathrm{LLE}}\left(\mathbf{x}^{\mathrm{E}, N}, \mathbf{x}^{\mathrm{R}, N}\right)
\end{aligned}
$$

$f_{\text {LLE }}(:)$ stands as a placeholder for thermodynamic phase equilibrium conditions. For $C$ components, there are $C$ phase equilibrium conditions per stage. For stage $P$ the pinch conditions

$$
\mathbf{x}^{\mathrm{R}, P} \cdot \dot{n}^{\mathrm{R}, P}=\mathbf{x}^{\mathrm{R},(P+1)} \cdot \dot{n}^{\mathrm{R},(P+1)}
$$

hold. Further, the material balances over multiple stages of the whole column yield two additional sets of independent equations, here:

$$
\begin{gathered}
\mathbf{x}^{\mathrm{R}, 1} \cdot \dot{n}^{\mathrm{R}, 1}-\mathbf{x}^{\mathrm{S}} \cdot \dot{n}^{\mathrm{S}}=\mathbf{x}^{\mathrm{R},(P+1)} \cdot \dot{n}^{\mathrm{R},(P+1)}-\mathrm{x}^{\mathrm{E}, P} \cdot \dot{n}^{\mathrm{E}, P} \\
\mathbf{x}^{\mathrm{R}, P} \cdot \dot{n}^{\mathrm{R}, P}-\mathbf{x}^{\mathrm{E},(P-1)} \cdot \dot{n}^{\mathrm{E},(P-1)}=\mathbf{x}^{\mathrm{F}} \cdot \dot{n}^{\mathrm{F}}-\mathbf{x}^{\mathrm{E}, N} \cdot \dot{n}^{\mathrm{E}, N} .
\end{gathered}
$$

The equation system is completed by the 12 summation conditions for the compositions of the 12 streams mentioned above:

$$
1=\sum_{i=1}^{C} x_{i}^{j} \quad \text { for } j \in\left\{\mathrm{F}, \mathrm{E}^{N}, \mathrm{R}^{N}, \mathrm{E}^{N-1}, \mathrm{R}^{P+1}, \mathrm{E}^{P}, \mathrm{R}^{P}, \mathrm{E}^{P-1}, \mathrm{R}^{2}, \mathrm{E}^{1}, \mathrm{R}^{1}, \mathrm{~S}\right\}
$$

In total these are $(9 C+12)$ equations with $(12 C+12)$ variables, leaving $3 C$ degrees of freedom.

\subsection{Slope diagram}

To study the solution of the equation system (Eqs. (1) - (10)) graphical methods can be used conveniently for up to $C=3$ components. Although not employed as part of the proposed formal methodology, the graphical slope curve method (SCM) which was presented recently in [14] is used here in the discussion and therefore described here briefly. For details, the reader is referred to [14]. The SCM introduces a so-called slope diagram, cf. Figure 2 right, which is based 
on a transformation of the well-known ternary diagram that is carried out such that straight lines in the ternary diagram are points in the slope diagram and vice versa. The transformation is done as follows: a Cartesian coordinate system is defined in the ternary diagram, as shown in Figure 2 left. Here, the $\hat{y}$-axis is pointing through the critical point, the $\hat{x}$-axis is pointing along the binary edge 2 - 3. In this coordinate system, each straight line in the ternary diagram (i.e. tie lines and material balance lines) is described by the linear equation:

$$
\hat{y}=m \hat{x}+b,
$$

where $m$ is the slope and $b$ is the $\mathrm{y}$-intercept of the line. In the slope diagram in Figure 2 right, $m$ is plotted over $b$.
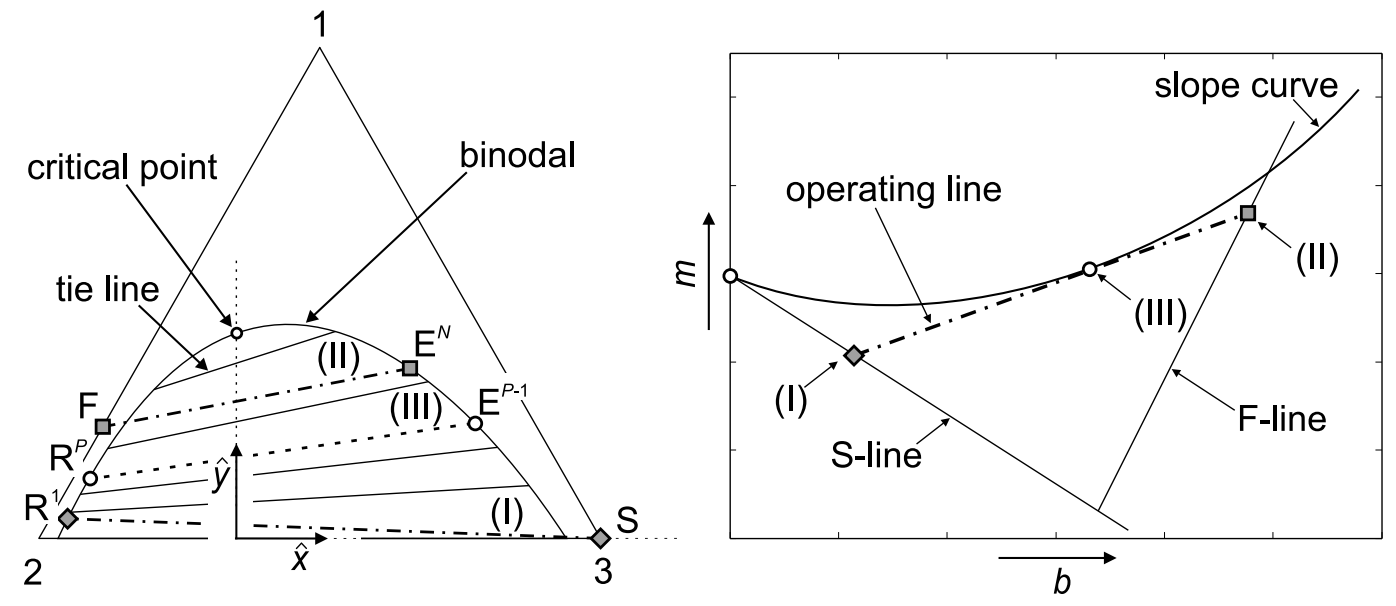

Figure 2: Transformation of the ternary diagram to the slope diagram. Left: ternary diagram with given $\mathrm{F}$ and $\mathrm{S}$ and one solution of the equation system (Eqs. (1) - (10)) leading to the lines (I), (II), and (III) connecting counter-current streams in the column, compare Figure 1. Right: slope diagram resulting from the transformation of the quantities given in the ternary diagram.

Every tie line of the LLE in the ternary diagram (c.f. Figure 2 left) is transformed to one point in the slope diagram where the collection of these points form a curve, the slope curve (c.f. Figure 2 right). Let us assume that 
a feasible column including molar flowrates and compositions of all streams is shown in Figure 1 and their compositions are given. All possible straight lines through the point marking the composition of the feed $\mathrm{F}$ in the ternary diagram are transformed to points in the slope diagram resulting in a collection of points that form a straight line, the so-called F-line (c.f. Figure 2 right). Analogously, the S-line is constructed that represents all straight lines in the ternary diagram that run through the point marking the composition of the solvent S. The intersection point of the S-line and F-line is called FSI-point. In the ternary diagram the FSI-point represents the line connecting F and S. In this example, the region enclosed by the F-line, the S-line, and the slope curve is non-convex.

We focus now on three specific lines in the ternary diagram (c.f. Figure 2 left) that connect compositions of two streams at the same height of the column:

(I) the line connecting $\mathrm{S}$ and $\mathrm{R}^{1}$

(II) the line connecting $\mathrm{F}$ and $\mathrm{E}^{N}$

(III) the line connecting $\mathrm{R}^{P}$ and $\mathrm{E}^{P-1}$ which is also a tie line.

In the slope diagram the following relations hold:

(I) is represented by a point on the S-line, since it is a straight line running through S

(II) is represented by a point on the F-line, since it is a straight line running through F

(III) is represented by a point on the slope curve, since from Eq. (7) follows: $\mathrm{E}^{P-1}=\mathrm{E}^{P}$ and therefore at the pinch the two streams $\mathrm{R}^{P}$ and $\mathrm{E}^{P-1}$ are in phases equilibrium (Eq. (5)).

In addition, the points (I), (II), and (III) have to be located on one straight line in the slope diagram, the so-called operating line. This is a consequence of the material balances Eq. (8) and Eq. (9), c.f. [14]. Figure 2 right shows an 
example of (I), (II), and (III), and the respective operating line. The geometric properties of a solution of the pinch equations in the slope diagram will be exploited later when the surrogate model is described.

\section{Surrogate model}

\subsection{Structure}

The requirements of the surrogate model are the following:

a) represent the equation system with the Eqs. (1) - (10)

b) consist only of process unit models that are typically available in common process simulators

c) employ thermodynamic models provided by the process simulator to avoid cumbersome implementation of property models

d) ability to return one non-ambiguous, physically meaningful solution out of the multiple solutions of the equation system (Eqs. (1) - (10)). 


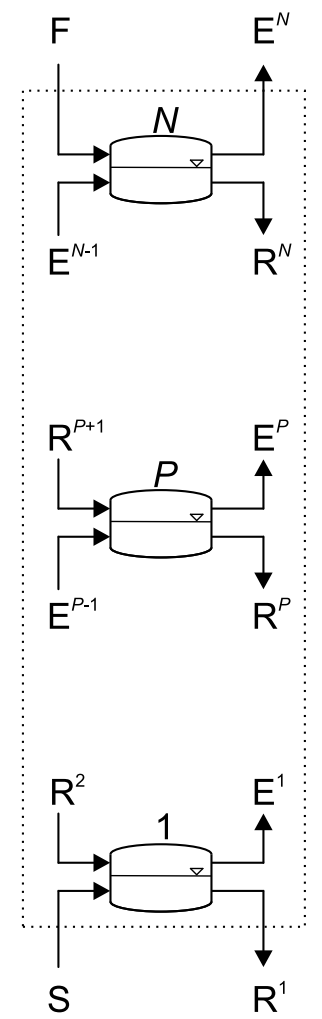

Figure 3: Surrogate model for the extraction column of infinite height. Three decanters are used. The streams entering and leaving the stages are connected by additional equations, see text.

The structure of the proposed surrogate model is shown in Figure 3. It consists of three decanters, a standard unit operation model available in every commercial process simulator. The decanters represent the top, the pinch, and the bottom stage of the column, respectively. The nomenclature is identical to Figure 1. Temperature and pressure of the decanters are specified to the ones of the column, which is assumed to be given. Each decanter model always contains a built-in material balance (Eqs. (1) - (3)) and phase equilibrium conditions (Eqs. (4) - (6)) which will therefore be fulfilled automatically for a convergent simulation. The summation equations (Eq. (10)) are also automatically fulfilled for all 12 streams since they are part of a flowsheet simulation. The 
remaining $3 C$ Eqs. (7) - (9), i.e. the pinch conditions and the material balances interconnecting the three decanters, are implemented via flowsheet specifications. After including the three decanters into the flowsheet and additionally specifying Eqs. (7) - (9) the simulation model exactly represents the Eqs. (1) (10) and the requirements a) - c) are met. As described in Section 2.1, the remain $3 C$ degrees of freedom, requiring $3 C$ additional specifications before the simulation problem is well-posed. $2 C$ specifications are typically obtained from the process task. E.g. the compositions $(C-1$ specifications each) and molar flow rates (1 specification each) of the streams $\mathrm{F}$ and $\mathrm{S}$ are given and one wants to calculate $\mathrm{E}^{N}$ and $\mathrm{R}^{1}$. Still, the simulation model has $C$ degrees of freedom left. One could say that the problem is under-specified and thus still not wellposed. Redepenning et al. [13] derive three additional constraints to eliminate the degrees of freedom using mathematical quanties like eigenvalues, but these quanties are not available without further ado in commercial process simulators. In the present work, two strategies are presented to obtain well-posed problems and their non-ambiguous solutions with standard simulator features. Both strategies require special problem cases, that are however quite common. These problem cases are:

a) the location of the pinch in the column is known and either at the top or the bottom

b) the number of components is limited to three.

For both cases, the solution strategy is outlined in the following.

\subsection{Pinch at the top or bottom}

In many problem formulations the location of the pinch is known a priori. For example, when the composition of $\mathrm{F}$ and $\mathrm{S}$ are given and the attainable minimum solute concentration in the raffinate is searched for. In this situation,

a large solvent stream is fed to the column, the pinch is on the solvent/raffinate side (solvent pinch [14]). Alternatively, one might want to know the minimum solvent flow rate to achieve a certain specification on the solute concentration 
in the raffinate. In this case, the pinch is located on the feed/extract side of the column (feed pinch [14]). If the location of the pinch is known, then $C$ additional specifications can be added:

$$
\mathbf{x}^{\mathrm{R},(P+1)}=\mathbf{x}^{\mathrm{R}, 2}
$$

for a solvent pinch, and

$$
\mathbf{x}^{\mathrm{E},(P-1)}=\mathbf{x}^{\mathrm{E},(N-1)}
$$

for a feed pinch, respectively. By introducing these additional specifications the problem becomes well-posed.

\subsection{Ternary systems}

Let us consider a ternary example. The example comprises the solute acetic acid (AA), the carrier water (W), and the solvent diisopropyl ether (DIPE) with a column operated at $25{ }^{\circ} \mathrm{C}$ and 1 bar. The LLE is calculated using the isoactivity criterion and the UNIQUAC model [16]. The UNIQUAC parameters are listed in the Supporting Information. The ternary diagram of the mixture is depicted in Figure 4, the slope curve of the LLE in Figure 5. The feed stream $\left(\dot{n}^{\mathrm{F}}=1 \mathrm{kmol} / \mathrm{h}, x_{\mathrm{W}}^{\mathrm{F}}=0.873 \mathrm{~mol} / \mathrm{mol}, x_{\mathrm{DIPE}}^{\mathrm{F}}=0 \mathrm{~mol} / \mathrm{mol}\right)$ and the solvent stream $\left(\dot{n}^{\mathrm{S}}=0.325 \mathrm{kmol} / \mathrm{h}, x_{\mathrm{DIPE}}^{\mathrm{S}}=1 \mathrm{~mol} / \mathrm{mol}\right)$ are given. Despite these specifications, three degrees of freedom remain, c.f. Section 2.1. The operating lines of three different solutions a), b), c) to the Eqs. (1) - (10) are shown in the ternary diagrams in Figure 4 and in the slope diagram in Figure 5. The task is to identify the physically meaningful amongst them. In the ternary diagrams (Figure 4) the difference between the three solutions is barely visible. In the slope diagram (Figure 5) each solution has an operating line connecting the Sline with the F-line and having at least one common point with the slope curve (cf. Section 2.2). Number and location of these common points give information on the type and location of the pinch(es). In case a) there is one internal pinch, in case b) there are two internal pinches, in case c) there is a solvent pinch. The theory behind the slope diagram [14] teaches that feasible operating lines lie entirely inside the region enclosed by the F-line, the S-line, and the slope curve. 
Thus, only solution a) is a feasible (physically meaningful) solution. Operating lines b) and c) are infeasible and have to be discarded. Operating line a) in Figure 5 has a feature that distinguishes it from the operating lines b) and c). It intersects the F-line and the S-line at points which are closer to the FSI-point when compared with the respective intersection points of the operating lines b) and c). The feasible solution a) minimizes the area $A$ of the triangle enclosed by the F-line, the S-line, and the operating line, i.e. the shaded area in Figure 5.
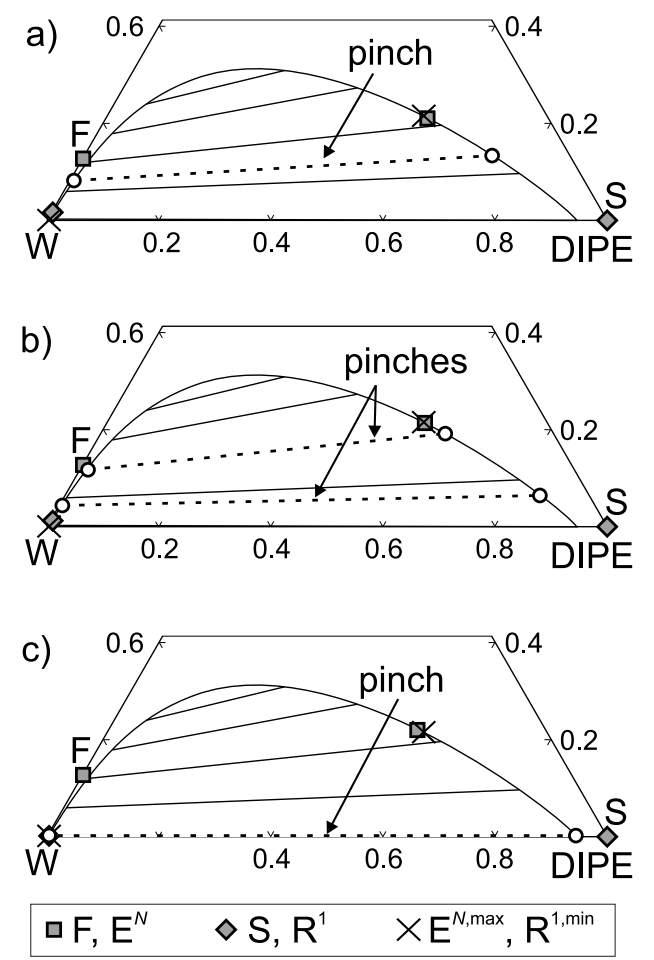

Figure 4: Ternary diagrams of the mixture acetic acid (AA, solute), water (W, carrier), diisopropylether (DIPA, solvent) at $25{ }^{\circ} \mathrm{C}$ and 1 bar. $\mathrm{F}$ is the feed and $\mathrm{S}$ the solvent. a), b), c) show the compositions of $\mathrm{E}^{N}$ and $R^{1}$ for different solutions of Eqs. (1) - (10). The corresponding operating lines for the cases a), b), and c) are given in the slope diagram in Figure 5. Concentrations in $\mathrm{mol} / \mathrm{mol}$. 


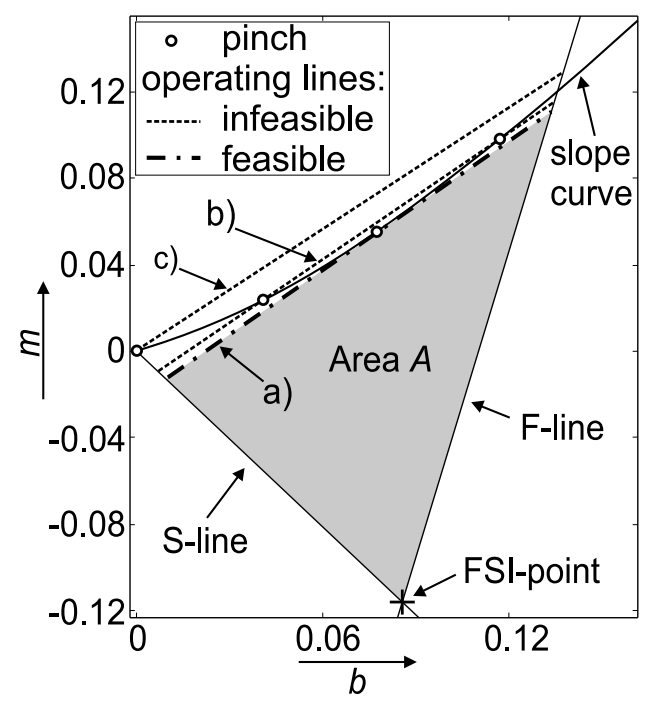

Figure 5: Slope diagram of the mixture acetic acid (AA, solute), water (W, carrier), diisopropylether (DIPA, solvent) at $25{ }^{\circ} \mathrm{C}$ and 1 bar for given $\mathrm{F}$ and $\mathrm{S}$. The operating lines a), b), and c) represent different solutions of the equations (1) - (10).

Based on this observation, the physically meaningful solution out of the multiple solutions of Eqs. (1) - (10) can be generally obtained by solving an optimization problem. As objective, one could minimize the area $A$ :

$$
\begin{array}{ll}
\min _{\substack{\mathrm{E}, P \\
\text { Solute }}} & A \\
\text { s.t. } & \text { Eqs. }(1)-(10) \\
& x_{\text {Solute }}^{\mathrm{E}, N} \leq x_{\text {Solute }}^{\mathrm{E}, N, \max } \\
& x_{\text {Solute }}^{\mathrm{R}, 1} \geq x_{\text {Solute }}^{\mathrm{R}, 1, \text { min }}
\end{array}
$$

As optimization variable the location of the pinch, expressed by solute concentration on one side of the pinch tie line (here $x_{\text {Solute }}^{\mathrm{E}, P}$ ), is used. Eqs. (1) - (10) are constraints preserving the original model equations of the surrogate model. The variables $x_{\text {Solute }}^{\mathrm{E}, N \text {,max }}$ and $x_{\text {Solute }}^{\mathrm{R}, 1, \min }$ denote the mole fraction of the solute in the extract at extremely low solvent flow rates and in the raffinate at extremely high solvent flow rates. These mole fractions must never be exceeded, no matter where the pinch is located [14]. 
In the process simulator, the compositions of all decanter streams are accessible and it is possible to calculate the area $A$ as a function of these compositions. However, this calculation is quite tedious, since it includes the coordinate transformation from the ternary diagram to the slope diagram. We therefore suggest using another objective function that is easier to access in the simulator. The optimization problem becomes:

$$
\begin{array}{ll}
\min _{x_{\text {Solute }}^{\mathrm{E}, P}} & \left(x_{\text {Solute }}^{\mathrm{E}, N}-x_{\text {Solute }}^{\mathrm{E}, 1}\right) \\
\text { s.t. } & \text { Eqs. }(1)-(10) \\
& x_{\text {Solute }}^{\mathrm{E}, N} \leq x_{\text {Solute }}^{\mathrm{E}, N, \max } \\
& x_{\text {Solute }}^{\mathrm{R}, 1} \geq x_{\text {Solute }}^{\mathrm{R}, 1, \min }
\end{array}
$$

Minimizing the difference $\left(x_{\text {Solute }}^{\mathrm{E}, N}-x_{\text {Solute }}^{\mathrm{E}, 1}\right)$ between the mole fractions of the solute in the extract phases at both ends of the column is equivalent to minimizing the area $A$. The reason for this equivalence is as follows: minimizing $A$ is equivalent to minimizing the distances of the triangle's upper corners to the FSI-point. If in the slope diagram the distances between the intersection points of the ends of an operating line (at F-line and S-line) to the FSI-point decrease, then the distance of the tie lines on which the column's products $\left(\mathrm{R}^{1}\right.$ and $\left.\mathrm{E}^{N}\right)$ are located in the ternary diagram decreases as well. The distance of the tie lines with $\mathrm{R}^{1}$ and $\mathrm{E}^{N}$ is measured here at one side of the binodal, expressed by the difference of the solute's concentration in the extract phases $\left(x_{\text {Solute }}^{\mathrm{E}, N}\right.$ and $\left.x_{\text {Solute }}^{\mathrm{E}, 1}\right)$. The constraints are identical to the ones described above.

Both formulations (Eq. (14) and Eq. (15)) also yield the correct solutions in case of feed or solvent pinches. This is shown in the next section that deals with numerical examples.

\subsection{Finding limiting product compositions}

In the constraints of Eq. (14) and (15) the limiting product compositions of the extract $\left(\mathbf{x}^{\mathrm{E}, N, \max }\right)$ and of the raffinate $\left(\mathrm{x}^{\mathrm{R}, 1, \mathrm{~min}}\right)$ for given feed streams are required. It is possible to determine them using the surrogate model, either 
in a pre-calculation step or by using a additional instances of the surrogate model in parallel. $\mathbf{x}^{\mathrm{E}, N, \max }$ equals the composition of $\mathbf{x}^{\mathrm{E}, N}$ when a feed pinch is specified, $\mathbf{x}^{\mathrm{R}, 1, \mathrm{~min}}$ equals the composition of $\mathbf{x}^{\mathrm{R}, 1}$ when a solvent pinch is specified. Thus, the limiting product compositions are obtained by specifying the surrogate model as described in Section 3.2

When no results but $\mathbf{x}^{\mathrm{E}, N, \max }$ or $\mathbf{x}^{\mathrm{R}, 1, \min }$ are needed from the surrogate model, it is sufficient to implement only parts of the surrogate model, namely the decanter in which the respective product occurs. For instance, if one wants to obtain $\mathrm{x}^{\mathrm{R}, 1, \mathrm{~min}}$ from the composition of $\mathrm{S}$, only the bottom-most decanter 1 in Figure 3 has to be implemented. In the spirit of Eq. (12), a pinch is specified in the decanter:

$$
\mathbf{x}^{\mathrm{R}, 1}=\mathbf{x}^{\mathrm{R}, 2}
$$

To obtain $\mathbf{x}^{\mathrm{E}, N, \max }$ from the composition of $\mathrm{F}$, the top decanter $N$ in Figure 3 has to be implemented with the specification:

$$
\mathbf{x}^{\mathrm{E}, N}=\mathbf{x}^{\mathrm{E},(N-1)} .
$$

In the example section it is shown how this technique can be used when simulating an entire flowsheet.

\section{Implementation and examples}

The implementation of the surrogate model in process simulation software is explained for the commercial software Aspen Plus [7]. In Aspen Plus specifications are realized using "design specs". For each design spec one manipulated variable has to be defined. Calculations of lengthy expression that are needed in the specifications are done with the "calculator block". Using design specs, in particular the Eqs. (7) - (9) and if applicable the Eqs. (12) and (13), are realized. A complete list of design specs for each example is given in the Supporting Information. 


\subsection{Pinch at the top or bottom}

The surrogate model is applied to a quaternary mixture with the carrier nheptane $(\mathrm{NH})$, the solvent propylene-carbonate $(\mathrm{PC})$ and two solutes o-xylene (OX) and toluene (TO). The column operating conditions are $25^{\circ} \mathrm{C}$ and 1 bar. The LLE is modeled with UNIQUAC and the parameters are listed in the Supporting Information. The solvent stream S is pure PC, the feed stream $\mathrm{F}$ is a ternary mixture of $\mathrm{NH}, \mathrm{OX}$ and $\mathrm{TO}\left(x_{O X}^{\mathrm{F}}=0.25 \mathrm{~mol} / \mathrm{mol}, x_{T O}^{\mathrm{F}}=\right.$ $0.35 \mathrm{~mol} / \mathrm{mol}$ ) with the molar flow rate $\dot{n}^{\mathrm{F}}=1 \mathrm{kmol} / \mathrm{h}$. Besides specifying the solvent flow rate $\left(\dot{n}^{\mathrm{S}}\right)$ the pinch location needs to be specified. The simulation results are the flow rate and compositions of the product streams $\left(\mathrm{E}^{N}\right.$ and $\left.\mathrm{R}^{1}\right)$. The results for different $\dot{n}^{\mathrm{S}}$ are shown in Figure 6 as lines which show the concentrations of the solutes in the raffinate and extract plotted over $\dot{n}^{\mathrm{S}}$. For the sake of readability, the concentrations of $\mathrm{NH}$ and $\mathrm{PC}$ in $\mathrm{E}^{\mathrm{N}}$ and $\mathrm{R}^{1}$ are not shown in Figure 6 but in the Supporting Information.
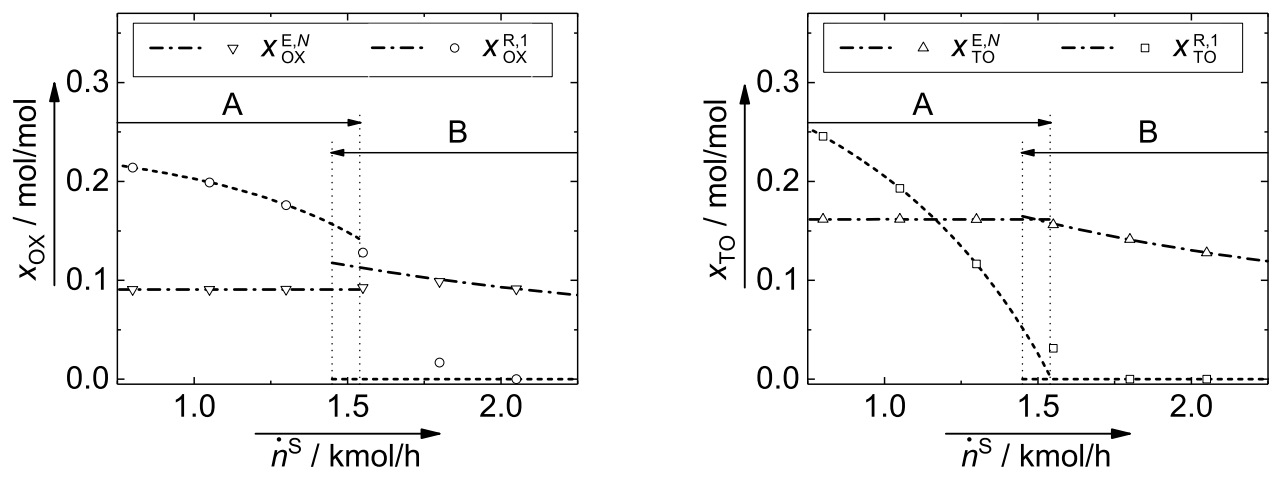

Figure 6: Results of the surrogate model (lines) for the quaternary mixture with the o-xylene (OX, solute), toluene ( $\mathrm{TO}$, solute), n-heptane (NH, carrier) and propylene-carbonate (PC, solvent) at $25{ }^{\circ} \mathrm{C}$ and 1 bar. Compositions of the solutes in the extract $\left(x_{O X}^{\mathrm{E}, N}, x_{T O}^{\mathrm{E}, N}\right)$ and raffinate $\left(x_{O X}^{\mathrm{R}, 1}, x_{T O}^{\mathrm{R}, 1}\right)$ are plotted over the solvent flow rate $\dot{n}^{\mathrm{S}}$. The symbols are obtained from an equilibrium stage column with 30 stages. A partially increased deviation between both models indicates that the occurrence of an internal pinch is very likely.

When assuming a feed pinch (Eq. (13)), a convergent simulation is obtained 
for values of the $\dot{n}^{\mathrm{S}}$ in the range A in Figure 6. When assuming a solvent pinch (Eq. (12)), convergent simulations are obtained in range B of the figure. Ranges $\mathrm{A}$ and $\mathrm{B}$ are overlapping. At least one of both assumptions has to be wrong in the overlapping range. Thus, the simulations might converge even if a wrong pinch location is assumed. This holds also outside the overlapping range as soon as an internal pinch in the column occurs. To verify the results of the surrogate model in this example, the simulations are repeated with an equilibrium stage model of the extraction column with $N=30$ stages at the same conditions. The results are shown as symbols in Figure 6. Obviously, the equilibrium stage model does not have infinitely many stages, but it still is able to show the weaknesses of the surrogate model. For $\dot{n}^{\mathrm{S}}<1.35 \mathrm{kmol} / \mathrm{h}$ and $\dot{n}^{\mathrm{S}}>1.95 \mathrm{kmol} / \mathrm{h}$, there is excellent agreement between surrogate model and equilibrium stage model indicating correct assumptions on the pinch location in these ranges. In the overlapping ranges and close to it, there are however obvious deviations which can be attributed to the occurrence of an internal pinch within an infinitely high column in that range. This example shows clearly the limitations of the surrogate model when there are more than three components. Only if the pinch location is correctly assumed, the results are reliable.

\subsection{Ternary systems}

In this example the optimization approach of Section 3.3 is used. The surrogate model reliably calculates the product compositions of the column independently from the location of the pinch. As example serves the system AA, $\mathrm{W}$, and DIPE. Process conditions and the compositions of $\mathrm{F}$ and $\mathrm{S}$ are already given in Section 3.3. The flowsheet is implemented as given in Figure 3. Using an sequential quadratic programming (SQP) optimization solver of Aspen Plus the problem of Eq. (15) is solved. The limiting product compositions needed in the last two constraints are pre-calculated as described in Section 3.4 in separate simulations. As in the previous example the compositions of $\mathrm{E}^{N}$ and $\mathrm{R}^{1}$ are calculated for different flow rates $\dot{n}^{\mathrm{S}}$. In Figure 7 the concentration of the solute in $\mathrm{E}^{N}\left(x_{\mathrm{AA}}^{\mathrm{E}, N}\right)$ and $\mathrm{R}^{1}\left(x_{\mathrm{AA}}^{\mathrm{R}, 1}\right)$ depending on $\dot{n}^{\mathrm{S}}$ are shown as lines. For $\dot{n}^{\mathrm{S}} \leq 0.28$ 
a feed pinch occurs, since $\mathrm{E}^{N}=\mathrm{E}^{N, \max }$. For $0.28<\dot{n}^{\mathrm{S}}<0.55$ neither $\mathrm{E}^{N, \max }$ nor $\mathrm{R}^{1, \min }$ is obtained. This behavior is caused by an internal pinch which is correctly determined by the surrogate model. For $\dot{n}^{\mathrm{S}} \geq 0.55$ a solvent pinch occurs $\left(\mathrm{R}^{1}=\mathrm{R}^{1, \mathrm{~min}}\right)$. To verify the results, selected product compositions are again also calculated with an equilibrium stage model with 30 stages at the same conditions (symbols in Figure 7). The agreement is excellent.

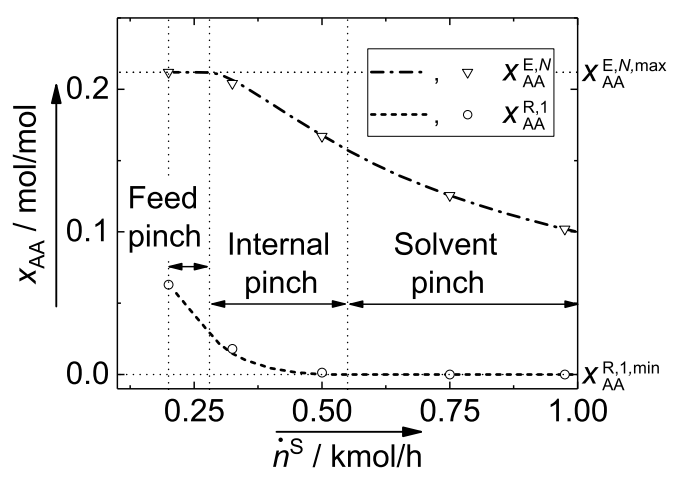

Figure 7: Results of the surrogate model for ternary mixtures (lines) shown for the example with acetic acid (AA, solute), water (carrier) and diisopropyl ether (solvent). The solute concentrations in the extract $\left(x_{\mathrm{AA}}^{\mathrm{E}, N}\right)$ and raffinate $\left(x_{\mathrm{AA}}^{\mathrm{R}, 1}\right)$ are plotted over $\dot{n}^{\mathrm{S}}$. The symbols are the results of an equilibrium stage model with 30 stages at the same conditions. The pinch locations are obtained from the surrogate model as well.

\subsection{Integration in process flowsheets}

Again the ternary mixture $\mathrm{W}+\mathrm{AA}+\mathrm{DIPE}$ is considered. The extraction column is modeled with the surrogate model for ternary mixtures using the optimization approach in Eq. (15) from Section 3.3. The column is integrated in a process with a solvent recovery by distillation and solvent recycle. The distillation column is modeled with the equilibrium stage model. The process flowsheet as implemented in Aspen Plus is shown in Figure 8. The VLE in the distillation column is calculated using a second independent set of UNIQUAC parameters (cf. Supporting Information). For describing the dimerization of AA in the vapor phase the dimerization constant given by Büttner and Maurer 
$[17]$ is used.

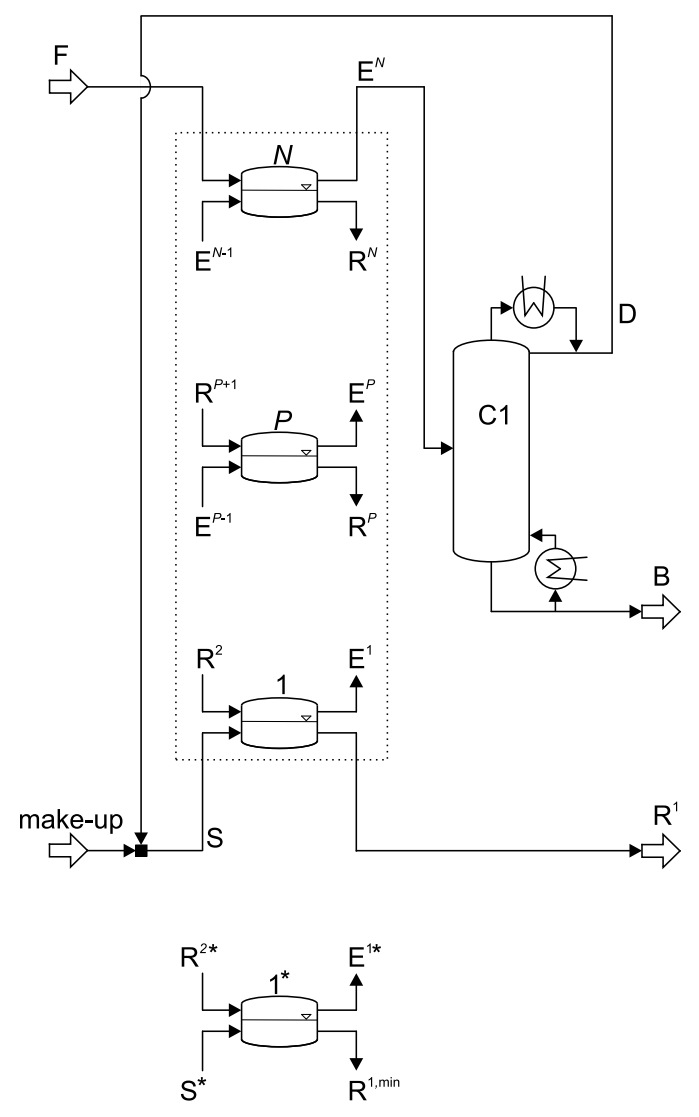

Figure 8: Flowsheet for the extraction-distillation process implemented in Aspen Plus. The dotted box is the surrogate model for the extraction column of infinite height. The decanter $1^{*}$ below the dotted box has to be simulated to determine the limiting raffiate composition $\left(\mathbf{x}^{\mathrm{R}, 1, \mathrm{~min}}\right)$.

The process feed $\mathrm{F}$ has the same composition and flow rate as described above and is given in Table 1 . The extract $\mathrm{E}^{N}$ is fed to the distillation column $\mathrm{C} 1$, where $\mathrm{AA}$ is obtained as bottom product $\mathrm{B}$. The distillate $\mathrm{D}$ of column $\mathrm{C} 1$ is mixed with a solvent make-up stream (pure DIPE) and fed as solvent $\mathrm{S}$ to the extraction column. The extraction column and the distillation column are operated at 1 bar. The extraction column is isothermal at $25^{\circ} \mathrm{C}$. The distillation 
column $\mathrm{C} 1$ has 20 equilibrium stages, $\mathrm{E}^{N}$ is fed to stage 10 . The purity of the bottom stream B is specified with $x_{\mathrm{AA}}^{\mathrm{B}}=0.999 \mathrm{~mol} / \mathrm{mol}$, obtained by varying the column's reflux ratio. The flow rate of stream B is set to $\dot{n}^{\mathrm{B}}=0.1 \mathrm{kmol} / \mathrm{h}$. Further the flow rate of DIPE in stream $\mathrm{S}$ is specified $\left(\dot{n}_{\mathrm{DIPE}}^{\mathrm{S}}=0.3 \mathrm{kmol} / \mathrm{h}\right)$. The flow rate of the make-up stream is the corresponding degree of freedom. Other specifications would be possible of course.

Since the composition of solvent stream $\mathrm{S}$ to the extraction column is unknown a-priori, the quantity $x_{\mathrm{DIPE}}^{\mathrm{R}, 1, \mathrm{~min}}$ which is required in Eq. (15) is calculated using an additional decanter $1^{*}$ as described in Section 3.4. Via a specification, the composition of stream $\mathrm{S}^{*}$ is set equal to the one of stream $\mathrm{S}$. The quantity $\mathrm{x}^{\mathrm{E}, N, \max }$ which is required in the other constraint of Eq. (15) is precalculated and does not change since the feed composition is constant.

The simulation is performed in a sequential-modular way, simultaneously using the SQP algorithm to solve the optimization problem. The simulation converged in 16 flowsheet iterations. Parts of the stream table and the composition of the limiting extract $\mathrm{E}^{N \text {,max }}$ are listed in Table 1 (for the entire stream table see in the Supporting Information). Further, the process streams are shown in the ternary diagram in Figure 9. As can be seen in the figure the column's distillate stream D is located in the LLE and could be fed to an additional decanter. This is not considered here and is out of the scope of this work.

Since $\mathrm{R}^{1} \neq \mathrm{R}^{1, \min }$ and $\mathrm{E}^{N} \neq \mathrm{E}^{N, \max }$ (cf. Table 1) the separation in the extraction column is controlled by the occurrence of an internal pinch. The location of the internal pinch is also obtained from the surrogate model $\mathrm{cf}$. $\mathrm{E}^{P}$ and $\mathrm{R}^{P}$ in Table 1 . 


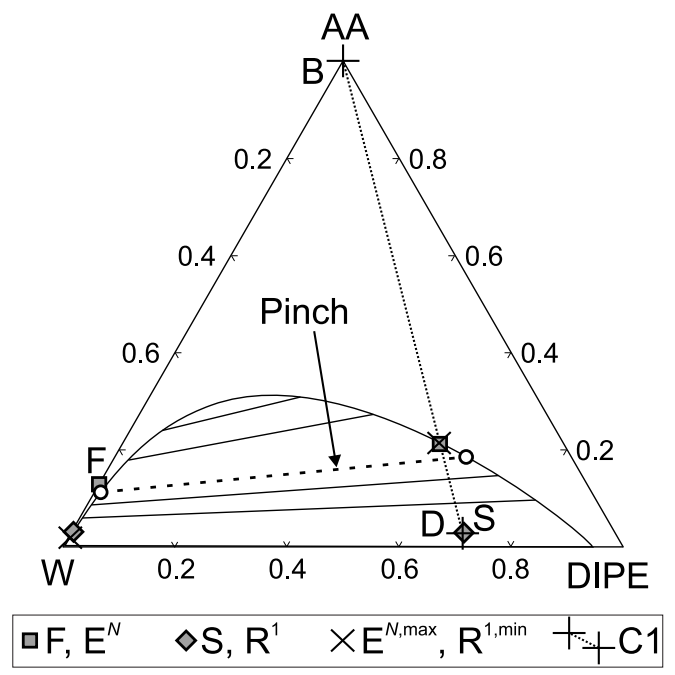

Figure 9: Process streams from Table 1 in a ternary diagram for the mixture acetic acid (AA, solute), water (W, carrier), diisopropylether (DIPA, solvent) at $25{ }^{\circ} \mathrm{C}$ and 1 bar. 


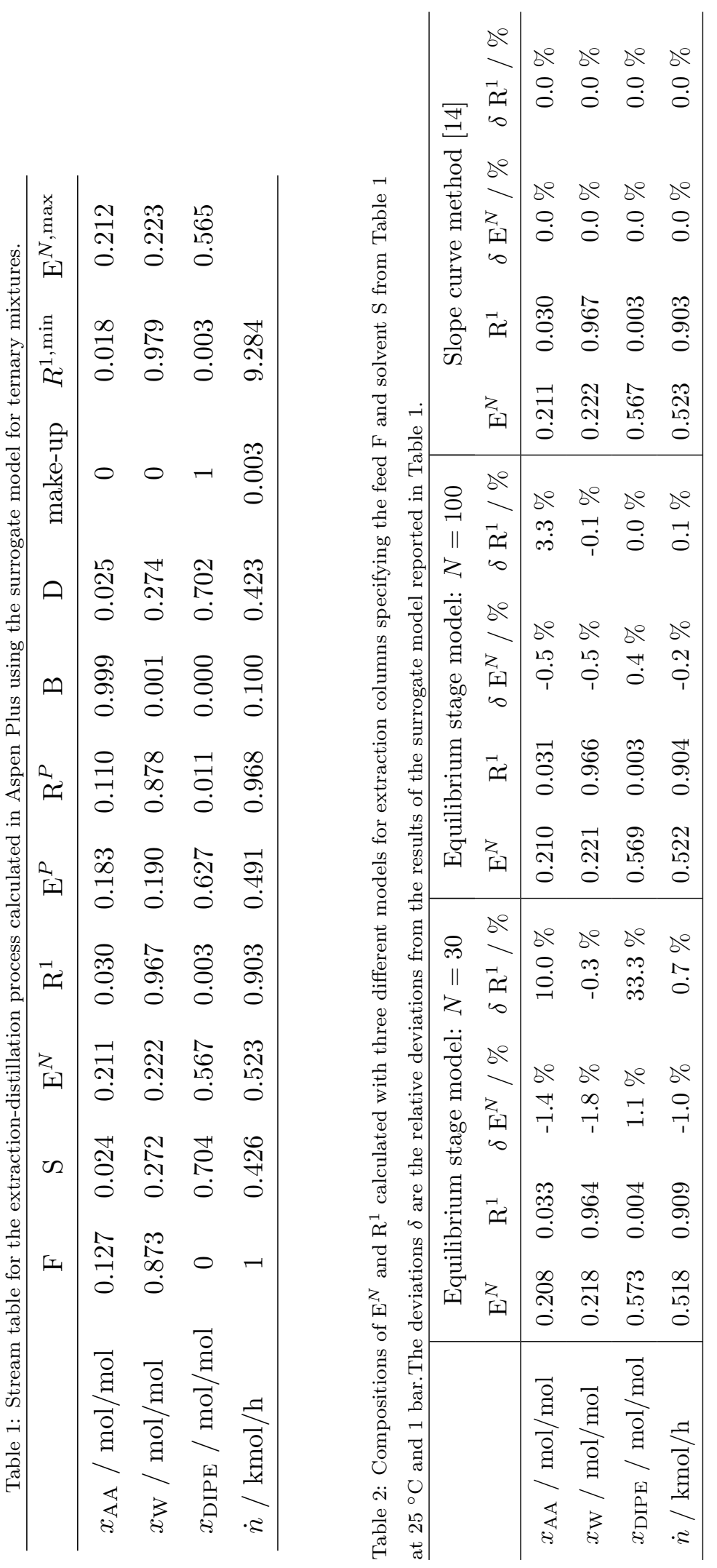


For the verification of the surrogate model, it is replaced by an equilibrium stage model with $N=30$ and also by one with $N=100$ stages. Further, raffinate and extract of the column are also determined with the SCM [14]. In all cases, the streams F and S are taken from Table 1. The results of all three alternatives are summarized in Table 2. The equilibrium stage column with 30 stages converged without further ado. For $N=100$ the number of stages had to be increased stepwise in cumbersome work. Furthermore, with $N=100$ even small perturbations of the solvent flow rate $\left(\Delta \dot{n}^{\mathrm{S}} \pm 0.01 \mathrm{kmol} / \mathrm{h}\right)$ lead to a divergent simulation in a re-run.

As expected the deviation of the surrogate model from the equilibrium stage model decreases with an increasing number of equilibrium stages. Nonetheless, even with $N=100$ stages there is a deviation between surrogate and equilibrium stage model. In contrast, the results of the SCM and the surrogate model are identical, representing both exactly an extraction column of an infinite height.

\section{Conclusion}

A surrogate model consisting of three decanters was proposed that enables a simple way to consider extraction columns of infinite height in commercial process simulation software. The combination of the surrogate model with other apparatuses in larger flowsheets is possible. For the surrogate model, no special software is necessary. By using the available tools (e.g. the built-in thermodynamic models) and standard apparatus models of the simulators, the surrogate model can be implemented easily. A comparison of the results of the surrogate model with the Slope Curve Method (SCM) shows that the surrogate model correctly presents a column of infinite height.

The model is applicable to systems of more than three components, if the pinch is knowingly located at one of the ends of the column. For ternary systems there are no restrictions regarding the pinch location and also internal pinches are described correctly.

The applications of the surrogate model are versatile. In conceptual process 
design it can be applied for feasibility studies or it can be used to determine the limiting product compositions. Furthermore, it can be used as extraction short-cut model in complex processes in which the extraction is not the main focus of the consideration.

\section{Acknowledgment}

The authors gratefully acknowledge financial support from "Stiftung RheinlandPfalz für Innovation" (Project no. 961-386261/1107). J.B. thanks BASF SE for financial support. 


\section{Nomenclature}

List of symbols

$\begin{array}{ll}b & \text { y-intercept } \\ C & \text { number of components } \\ m & \text { slope } \\ N & \text { number of stages } \\ \dot{n}^{j} & \text { molar flow rate stream } j \\ P & \text { pinch stage } \\ \hat{x} & \text { coordinate } \\ x_{i}^{j} & \text { mole fraction component } i \text { in stream } j \\ \mathbf{x}^{j} & \text { molar composition vector stream } j \\ \hat{y} & \text { coordinate }\end{array}$

Abbreviations
AA acetic acid
B bottom stream
cp critical point
D distillate stream
DIPE diisopropyl ether
E extract stream
$\mathrm{E}^{N, \max }$ limiting extract stream
F feed stream
LLE liquid-liquid equilibrium
NH n-heptane
OX o-xylene
PC propylene-carbonate
$\mathrm{R} \quad$ raffinate stream
$\mathrm{R}^{1, \text { min }} \quad$ limiting raffinate stream
S solvent stream
TO toluene
SCM slope curve method
VLE vapor-liquid equilibrium
W water
25 


\section{References}

[1] J. G. Stichlmair, J. R. Fair, Distillation: Principles and Practice, 1st Edition, Wiley-VCH, New York, USA, 1998.

[2] F. B. Petlyuk, Distillation Theory and Its Application to Optimal Design of Separation Units, Cambridge University Press, Cambridge, 2004.

URL http://ebooks.cambridge.org/ref/id/CB09780511547102

[3] O. Ryll, S. Blagov, H. Hasse, $\infty / \infty$-Analysis of Homogeneous Distillation Processes, Chemical Engineering Science 84 (0) (2012) 315 - 332. doi:10.1016/j.ces.2012.08.018.

[4] M. Minotti, M. F. Doherty, M. F. Malone, A Geometric Method for the Design of Liquid Extractors, Industrial \& Engineering Chemistry Research 35 (8) (1996) 2672-2681. doi:10.1021/ie950687+.

[5] M. Doherty, M. Malone, Conceptual Design of Distillation Systems, 1st Edition, McGraw-Hill, New York, USA, 2001.

[6] S. G. Levy, D. B. Van Dongen, M. F. Doherty, Design and Synthesis of Homogeneous Azeotropic Distillations. 2. Minimum Reflux Calculations for Non Industrial \& Engineering Chemistry Fundamentals 24 (4) (1985) 463-474. doi:10.1021/i100020a011.

URL http://dx.doi.org/10.1021/i100020a011

[7] AspenPlus V8.4, Aspen Technology, Inc., Cambridge, USA, 2013.

[8] J. Bausa, R. v. Watzdorf, W. Marquardt, Shortcut Methods for Nonideal Multicomponent Distillation: I. Simple Columns, AIChE Journal $44 \quad(10) \quad$ (1998) 2181-2198, testaa. doi:10.1002/aic.690441008.

URL http://dx.doi.org/10.1002/aic.690441008

[9] R. von Watzdorf, J. Bausa, W. Marquardt, Shortcut Methods for Nonideal Multicomponent Distillation: 2. Complex Columns, 
AIChE Journal 45 (8) (1999) 1615-1628. doi:10.1002/aic.690450803.

URL http://onlinelibrary.wiley.com/doi/10.1002/aic.690450803/abstract

[10] O. Ryll, S. Blagov, H. Hasse, $\infty / \infty$-Analysis of Heterogeneous Distillation Processes, Chemical Engineering Science 104 (0) (2013) 374 - 388. doi:10.1016/j.ces.2013.09.012.

[11] O. Ryll, S. Blagov, H. Hasse, Thermodynamic Analysis of Reaction-Distillation Processes Based on Piecewise Linear Models, Chemical Engineering Science 109 (0) (2014) $284 \quad$ - 295. doi:10.1016/j.ces.2014.01.024.

[12] M. Minotti, M. F. Doherty, M. F. Malone, Economic Tradeoffs for Extraction Systems, Chemical Engineering Research and Design 76 (3) (1998) 361 - 367. doi:http://dx.doi.org/10.1205/026387698524785.

[13] C. Redepenning, S. Recker, W. Marquardt, Pinch-Based Shortcut Method for the Conceptual Design of Isothermal Extraction Columns, AIChE Journal 63 (4) (2017) 1236-1245. doi:10.1002/aic.15523.

[14] J. Burger, M. Kaul, H. Hasse, Slope Curve Method for the Analysis of Separations in Extraction Columns of Infinite Height, Chemical Engineering Science 143 (2016) 105-113. doi:10.1016/j.ces.2015.12.008.

[15] J. Bonet, V. Plesu, A.-E. Bonet-Ruiz, A. Tuluc, J. Llorens, P. Iancu, Minimum Solvent Flow Rate for Counter-Current Liquid-Liquid Extraction Columns, Pres 2014, 17th Conference on Process Integration, Modelling and Optimisation for Energy Saving and Pollution Reduction, Pts 1-3 39 (2014) 1771-1776. doi:10.3303/CET1439296.

[16] D. S. Abrams, J. M. Prausnitz, Statistical Thermodynamics of Liquid Mixtures: A New Expression for the Excess Gibbs Energy of Partly or Completely Miscible Systems, AIChE Journal 21 (1) (1975) 116-128. doi:10.1002/aic.690210115. 
[17] R. Büttner, G. Maurer, Dimerisierung einiger organischer Säuren in der Gasphase, Berichte der Bunsengesellschaft für physikalische Chemie 87 (10) (1983) 877-882. doi:10.1002/bbpc.19830871010.

[18] A. B. S. H. Salem, E. Z. Hamad, M. A. Al-Naafa, Quaternary Liquid-Liquid Equilibrium of n-Heptane-Toluene-o-Xylene-Propylene Carbonate, Industrial \& Engineering Chemistry Research 33 (3) (1994) 689-692. doi:10.1021/ie00027a029.

URL http://dx.doi.org/10.1021/ie00027a029

[19] D. F. Othmer, S. J. Silvis, A. Spiel, Composition of Vapors from Boiling Binary Solutions: Pressure Equilibrium Still for Studying WaterAcetic Acid System, Industrial \& Engineering Chemistry 44 (8) (1952) 1864-1872. doi:10.1021/ie50512a041.

[20] I. Brown, A. Ewald, Liquid-Vapour Equilibria Equilibria .1. The Systems Carbon Tetrachloride-Cyclohexane and Water - Acetic Acid, Australian Journal of Scientific Research Series A-Physical Sciences 3 (1950) 306-323. 


\section{Supporting Information}

\section{Property Data}

The UNIQUAC parameters for modeling of the liquid-liquid equilibrium (LLE) and the vapor-liquid equilibrium (VLE) are listed in Table 3 and Table 4. For the VLE the dimerization of the acetic acid in the vapor phase is considered by the dimerization constant given by [17].

Table 3: UNIQUAC pure component parameters used for modeling the liquid-liquid equilibrium and the vapor-liquid equilibrium in the current work.

\begin{tabular}{lcc}
\hline Component & $r$ & $q$ \\
\hline Acetic acid [7] & 2.19512 & 2.072 \\
Diisopropyl ether [7] & 4.74225 & 4.088 \\
Water [7] & 0.9200 & 1.400 \\
n-Heptane [18] & 5.1742 & 4.396 \\
Propylene-carbonate [18] & 3.2815 & 2.736 \\
o-Xylene [18] & 4.6578 & 3.536 \\
Toluene [18] & 3.9228 & 2.968 \\
\hline
\end{tabular}


Table 4: Binary UNIQUAC parameters $\left(\tau_{i j}=b_{i j} / T\right)$ used for modeling the liquid-liquid equilibrium and the vapor-liquid equilibrium in the current work.

\begin{tabular}{llcc}
\hline Component $i$ & Component $j$ & $b_{i j} / \mathrm{K}$ & $b_{j i} / \mathrm{K}$ \\
\hline Liquid-liquid equilibrium: & & \\
Acetic acid & Diisopropyl ether [7] & 106.34 & -45.707 \\
Water & Acetic acid [7] & -73.444 & 219.660 \\
Water & Diisopropyl ether [7] & -86.665 & -602.34 \\
n-Heptane & Toluene [18] & -347.16 & 218.52 \\
n-Heptane & Propylene-carbonate [18] & -1266.6 & 15.667 \\
Toluene & Propylene-carbonate [18] & -190.06 & 22.65 \\
n-Heptane & o-Xylene [18] & 11.24 & 13.184 \\
o-Xylene & Propylene-carbonate [18] & -190.31 & 22.1 \\
\hline Vapor-liquid equilibrium: & & \\
Acetic acid & Diisopropyl ether [7] & 134.3754 & -397.9367 \\
Water & Acetic acid a) & -585.41 & 257.91 \\
Water & Diisopropyl ether [7] & -29.592 & -774.7116 \\
\hline a) & & & \\
\hline
\end{tabular}

a) fitted to experimental data from [19] and [20] using the dimerization constant given by [17]: $\ln K_{\mathrm{AA}}=-19.1001+7928.7 /(T / K)$.

\section{Pinch at the top or bottom: o-xylene, toluene, n-heptane, and propylene-carbonate}

In Figure 10 the results for the quaternary mixture o-xylene (OX, solute) and toluene (TO, solute), n-heptane (NH, carrier) and propylene-carbonate (PC, solvent) are given. The compositions of the components NH and PC are plotted over $\dot{n}^{\mathrm{S}}$. 

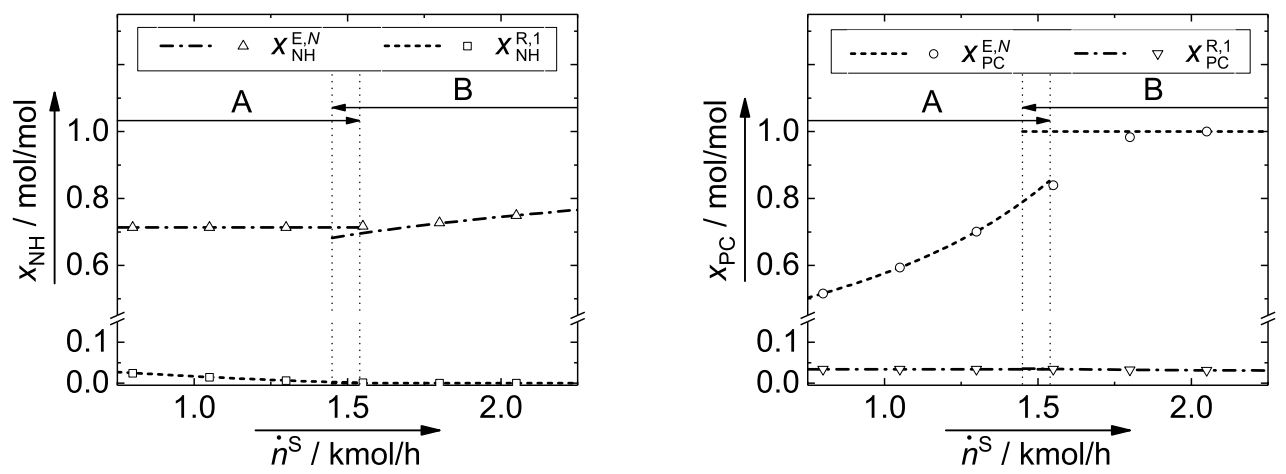

Figure 10: Results of the surrogate model (lines) for the quaternary mixture with o-xylene (OX, solute) and toluene (TO, solute), n-heptane ( $\mathrm{NH}$, carrier) and propylene-carbonate (PC, solvent) at $25^{\circ} \mathrm{C}$ and 1 bar. Compositions of $\mathrm{NH}$ and $\mathrm{PC}$ in the extract $\left(x_{N H}^{\mathrm{E}, N}, x_{P C}^{\mathrm{E}, N}\right)$ and raffinate $\left(x_{N H}^{\mathrm{R}, 1}, x_{P C}^{\mathrm{R}, 1}\right)$ are plotted over the solvent flow rate $\dot{n}^{\mathrm{S}}$. The symbols are obtained from an equilibrium stage column with 30 stages. A partially increased deviation between both models indicates that the occurrence of an internal pinch is very likely.

\section{Design specs in Aspen Plus}

In this chapter, the implementation of the surrogate model in Aspen Plus is described in detail for a ternary mixture with solute (1), carrier (2) and solvent (3).

\subsection{Finding limiting product compositions}

To determine the limiting product compositions for either product stream $\left(\mathrm{E}^{N, \max }\right.$ or $\left.\mathrm{R}^{1, \min }\right)$ one decanter is necessary. Here the design specs to obtain $\mathrm{E}^{N, \text { max }}$ are described for a ternary mixture (cf. Table 5). The nomenclature of the decanter $N^{*}$ is given in Figure 11. The extract composition of an arbitrary tie line can be used as starting value for $E^{(N-1)^{*}}$. 


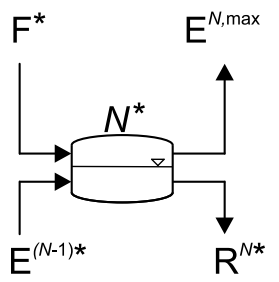

Figure 11: Decanter $N^{*}$ to determine $\mathbf{x}^{\mathrm{E}, N, \max }$.

Table 5: Desing specs to determine the limiting extract composition $\mathrm{E}^{N, \max }$.

\begin{tabular}{ll}
\hline Specification & \multicolumn{1}{c}{ vary } \\
\hline $0=x_{1}^{\mathrm{E},(N-1)^{*}}-x_{1}^{\mathrm{E}, N, \max }$ & $\dot{n}_{1}^{\mathrm{E},(N-1)^{*}}$ \\
$0=x_{2}^{\mathrm{E},(N-1)^{*}}-x_{2}^{\mathrm{E}, N, \max }$ & $\dot{n}_{2}^{\mathrm{E},(N-1)^{*}}$ \\
\hline
\end{tabular}

\subsection{Pinch at the top or bottom}

The design specs (Table 6) are given for the case that a feed pinch occurs. Therein, the streams $\Delta^{N}$ and $\Delta^{1}$ are virtual streams that are calculated as the difference of the two counter-current streams at both ends of the column respectively. See Table 7 for details. The calculations in Table 7 are implemented in Aspen Plus using the calculator block. The extract composition of an arbitrary tie line can be used as starting value for $E^{(P-1)}$.

Table 6: Desing specs of the surrogate model for a given pinch location. Here a feed pinch is assumed.

\begin{tabular}{lc}
\hline Specification & vary \\
\hline $0=x_{1}^{\mathrm{E},(P-1)}-x_{1}^{\mathrm{E}, P}$ & $\dot{n}_{1}^{\mathrm{E},(P-1)}$ \\
$0=x_{2}^{\mathrm{E},(P-1)}-x_{2}^{\mathrm{E}, P}$ & $\dot{n}_{2}^{\mathrm{E},(P-1)}$ \\
\hline $0=x_{1}^{\mathrm{E},(P-1)}-x_{1}^{\mathrm{E},(N-1)}$ & $\dot{n}_{1}^{\mathrm{E},(N-1)}$ \\
$0=x_{2}^{\mathrm{E},(P-1)}-x_{2}^{\mathrm{E},(N-1)}$ & $\dot{n}_{3}^{\mathrm{E},(N-1)}$ \\
\hline $0=\dot{n}_{1}^{\Delta, \mathrm{N}}-\dot{n}_{1}^{\Delta, 1}$ & $\dot{n}^{\mathrm{E},(N-1)}$ \\
$0=\dot{n}_{2}^{\Delta, \mathrm{N}}-\dot{n}_{2}^{\Delta, 1}$ & $\dot{n}_{1}^{\mathrm{R}, 2}$ \\
$0=\dot{n}_{3}^{\Delta, \mathrm{N}}-\dot{n}_{3}^{\Delta, 1}$ & $\dot{n}_{3}^{\mathrm{R}, 2}$ \\
\hline
\end{tabular}


Table 7: Calculator Block to determine the $\Delta$ streams of decanter 1 and $N$.

$$
\begin{aligned}
& \dot{n}_{1}^{\Delta, \mathrm{N}}=x_{1}^{\mathrm{F}} \cdot \dot{n}^{\mathrm{F}}-x_{1}^{\mathrm{E}, N} \cdot \dot{n}^{\mathrm{E}, N} \\
& \dot{n}_{2}^{\Delta, \mathrm{N}}=x_{2}^{\mathrm{F}} \cdot \dot{n}^{\mathrm{F}}-x_{2}^{\mathrm{E}, N} \cdot \dot{n}^{\mathrm{E}, N} \\
& \dot{n}_{3}^{\Delta, \mathrm{N}}=x_{3}^{\mathrm{F}} \cdot \dot{n}^{\mathrm{F}}-x_{3}^{\mathrm{E}, N} \cdot \dot{n}^{\mathrm{E}, N} \\
& \dot{n}_{1}^{\Delta, 1}=x_{1}^{\mathrm{R}, 1} \cdot \dot{n}^{\mathrm{R}, 1}-x_{1}^{\mathrm{S}} \cdot \dot{n}^{\mathrm{S}} \\
& \dot{n}_{2}^{\Delta, 1}=x_{2}^{\mathrm{R}, 1} \cdot \dot{n}^{\mathrm{R}, 1}-x_{2}^{\mathrm{S}} \cdot \dot{n}^{\mathrm{S}} \\
& \dot{n}_{3}^{\Delta, 1}=x_{3}^{\mathrm{R}, 1} \cdot \dot{n}^{\mathrm{R}, 1}-x_{3}^{\mathrm{S}} \cdot \dot{n}^{\mathrm{S}} \\
& \hline
\end{aligned}
$$

\subsection{Ternary systems}

For ternary systems an optimization procedure is applied. The specification of the pinch location is not necessary.

For the optimization problem in Eq. (15) of the manuscript the objective function is $x_{\text {Solute }}^{\mathrm{E}, N}-x_{\text {Solute }}^{\mathrm{E}, 1}$. The design specs for the implementation in Aspen Plus are given in Table 8 . The virtual $\Delta$ streams represent the difference of the counter-current streams of the column at different heights. The $\Delta$ streams of the decanters $1, P$, and $N$ are calculated again with a calculator block (cf. Table 9). The compositions of an arbitrary tie line are used as starting value for $R^{P+1}$ and $E^{P}$. If the model does not converge instantly it was found helpful to reinitialize the simulation while varying the starting value for $R^{P+1}$ and $E^{P}$. 
Table 8: Design specs of the surrogate model for ternary systems.

\begin{tabular}{lc}
\hline Specification & vary \\
\hline $0=x_{2}^{\mathrm{E},(P-1)}-x_{2}^{\mathrm{E}, P}$ & $\dot{n}_{2}^{\mathrm{E},(P-1)}$ \\
$0=x_{1}^{\mathrm{R},(P+1)}-x_{1}^{\mathrm{R}, P}$ & $\dot{n}_{1}^{\mathrm{R},(P+1)}$ \\
$0=x_{3}^{\mathrm{R},(P+1)}-x_{3}^{\mathrm{R}, P}$ & $\dot{n}_{3}^{\mathrm{R},(P+1)}$ \\
\hline $0=\dot{n}_{3}^{\Delta, 1}-\dot{n}_{3}^{\Delta, \mathrm{P}}$ & $\dot{n}^{\mathrm{E},(P-1)}$ \\
$0=\dot{n}_{1}^{\Delta, \mathrm{P}}-\dot{n}_{1}^{\Delta, 1}$ & $\dot{n}_{1}^{\mathrm{R}, 2}$ \\
$0=\dot{n}_{2}^{\Delta, 1}-\dot{n}_{2}^{\Delta, \mathrm{P}}$ & $\dot{n}_{2}^{\mathrm{R}, 2}$ \\
$0=\dot{n}_{1}^{\Delta, \mathrm{P}}-\dot{n}_{1}^{\Delta, \mathrm{N}}$ & $\dot{n}_{1}^{\mathrm{E},(N-1)}$ \\
$0=\dot{n}_{3}^{\Delta, \mathrm{P}}-\dot{n}_{3}^{\Delta, \mathrm{N}}$ & $\dot{n}_{3}^{\mathrm{E},(N-1)}$ \\
$0=\dot{n}_{2}^{\Delta, \mathrm{P}}-\dot{n}_{2}^{\Delta, \mathrm{N}}$ & $\dot{n}^{\mathrm{R},(P+1)}$ \\
\hline
\end{tabular}

Table 9: Calculator block to determine the $\Delta$ streams of decanter $1, P$, and $N$.

$$
\begin{aligned}
& \dot{n}_{1}^{\Delta, \mathrm{N}}=x_{1}^{\mathrm{F}} \cdot \dot{n}^{\mathrm{F}}-x_{1}^{\mathrm{E}, N} \cdot \dot{n}^{\mathrm{E}, N} \\
& \dot{n}_{2}^{\Delta, \mathrm{N}}=x_{2}^{\mathrm{F}} \cdot \dot{n}^{\mathrm{F}}-x_{2}^{\mathrm{E}, N} \cdot \dot{n}^{\mathrm{E}, N} \\
& \dot{n}_{3}^{\Delta, \mathrm{N}}=x_{3}^{\mathrm{F}} \cdot \dot{n}^{\mathrm{F}}-x_{3}^{\mathrm{E}, N} \cdot \dot{n}^{\mathrm{E}, N} \\
& \dot{n}_{1}^{\Delta, \mathrm{P}}=x_{1}^{\mathrm{R},(P+1)} \cdot \dot{n}^{\mathrm{R},(P+1)}-x_{1}^{\mathrm{E}, P} \cdot \dot{n}^{\mathrm{E}, P} \\
& \dot{n}_{2}^{\Delta, \mathrm{P}}=x_{2}^{\mathrm{R},(P+1)} \cdot \dot{n}^{\mathrm{R},(P+1)}-x_{2}^{\mathrm{E}, P} \cdot \dot{n}^{\mathrm{E}, P} \\
& \dot{n}_{3}^{\Delta, \mathrm{P}}=x_{3}^{\mathrm{R},(P+1)} \cdot \dot{n}^{\mathrm{R},(P+1)}-x_{3}^{\mathrm{E}, P} \cdot \dot{n}^{\mathrm{E}, P} \\
& \dot{n}_{1}^{\Delta, 1}=x_{1}^{\mathrm{R}, 1} \cdot \dot{n}^{\mathrm{R}, 1}-x_{1}^{\mathrm{S}} \cdot \dot{n}^{\mathrm{S}} \\
& \dot{n}_{2}^{\Delta, 1}=x_{2}^{\mathrm{R}, 1} \cdot \dot{n}^{\mathrm{R}, 1}-x_{2}^{\mathrm{S}} \cdot \dot{n}^{\mathrm{S}} \\
& \dot{n}_{3}^{\Delta, 1}=x_{3}^{\mathrm{R}, 1} \cdot \dot{n}^{\mathrm{R}, 1}-x_{3}^{\mathrm{S}} \cdot \dot{n}^{\mathrm{S}}
\end{aligned}
$$

\section{Stream table process simulation}

The complete stream table of the studied distillation-extraction process is given in Table 10. 


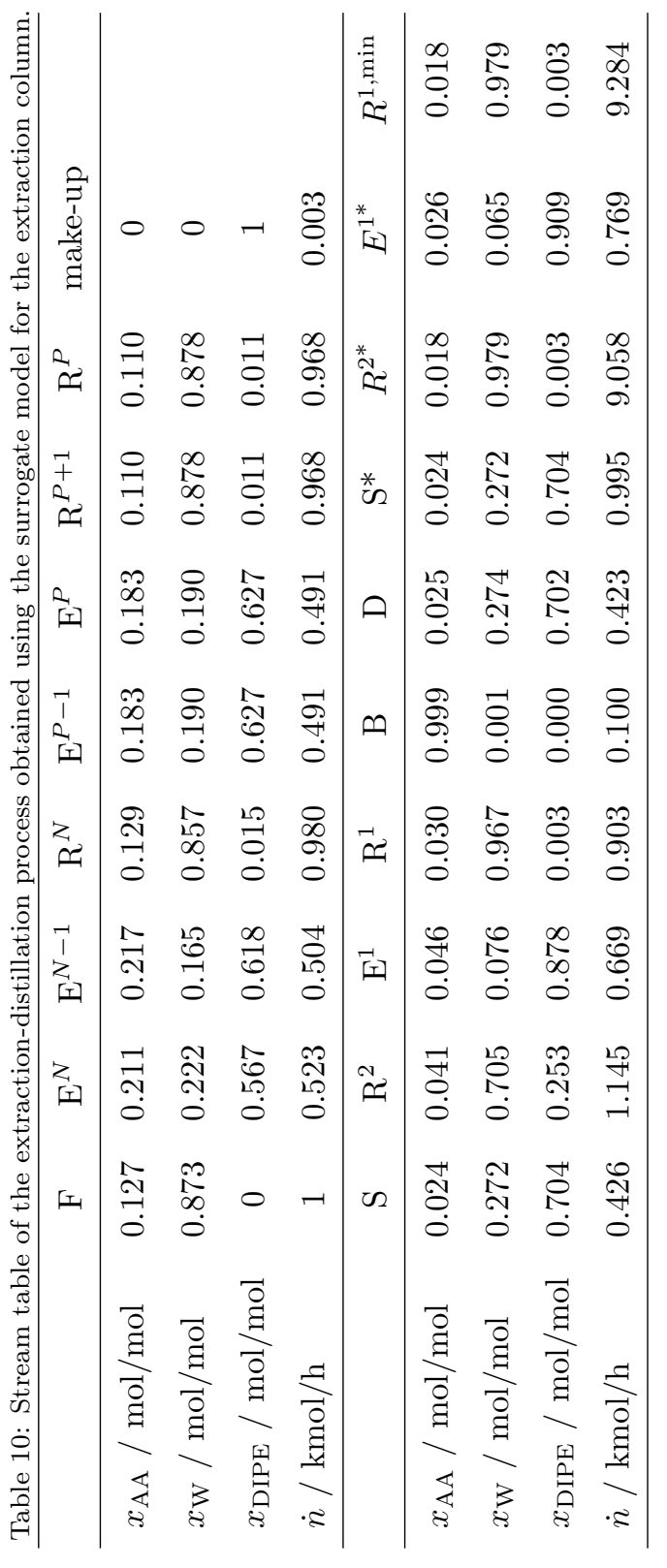

